\title{
RETARD DE FLUORESCENCE RETINIENNE COMME CRITERE DE MORT
}

\author{
Hans Petter Brinck, Lorents Gran et John Ludvig Larsen
}

IL N'EST PAS RARE, à la suite d'un traumatisme crânien ou d'une resuscitation, de voir le malade demeurer profondément comateux par suite de mort cérébrale ; le reste de l'organisme peut cependant demeurer longtemps en bon état pour peu que la ventilation et la circulation soient assistées. En ces circonstances, la circulation intra-crânienne est ralentie et s'arrête, et les tissus nerveux cérébraux devenus complètement ischémiques se nécrosent. On est alors en présence d'un coma dépassé car le malade est irrévocablement mort ; la continuation du traitement devient par conséquent difficilement défendable, tant du point de vue médical que des points de vue humain et moral.

Le diagnostic de cet état repose principalement sur les données de l'examen neurologique et ophtalmologique. En cas de doute, seule l'angiographie cérébrale peut démontrer l'arrêt de la circulation intra-crânienne. Malheureusement, le nombre d'hôpitaux dotés de l'équipement angiographique nécessaire est limité (du moins en Norvège) alors que le traitement intensif avec respirateur est praticable à peu près dans tous les centres hospitaliers.

La fluoroscopie rétinienne peut représenter une méthode à la fois simple et rapide, applicable dans tous les hôpitaux, pour étudier l'état de la circulation intra-crânienne. Le but de ce travail est d'évaluer la fiabilité de cette méthode en comparant les résultats obtenus à ceux de l'angiographie cérébrale.

\section{MéTHOde}

La fluoroscopie rétinienne avec mesure du temps de circulation bras-rétine, a été proposée en 1961 pour le diagnostic de sténose ou d'occlusion au niveau des grandes artères du cou. Nous avons suivi le mème procédé avec quelques modifications : le colorant utilisé est la fluorescéine en solution sodique à 10 pour cent. Chez l'adulte, on injecte $5 \mathrm{ml}$ de cette solution dans une veine du pli du coude ; le temps exact a été mesurẻ au chronographe dès la fin de l'injection

Service Ophtalmologique, Service de Réanimation et Service de Radiologie, Clinque Universitaire Haukeland Sykehus, Bergen, Norvège.

Canad. Anaesth. Soc. J., vol. 26, no. 4, July 1979 intra-veineuse, jusqu'au premier signe de fluorescence dans la rétine. Celle-ci est observée sous ophtalmoscope muni d'un filtre bleu spécial.

L'examen de nos malades comateux comporte:

Premièrement, un bilan clinique avec évaluation de:

(a) l'état général:

la température

le rythme cardiaque

la tension artérielle sanguine

le débit urinaire

la respiration propre

(b) l'état neurologique:

le niveau de conscience

les réflexes des nerfs crâniaux

la réaction à la stimulation douloureuse ou aspiration des bronches

(c) l'état ophtalmologique:

les mouvements spontanés des yeux

la réaction des pupilles à la lumière

la sensibilité de la cornée

l'état du fond d'oeil

Deuxièmement, la fluroscopie rétinienne telle qu'indiquée ci-haut.

Troisièmement, une ciné-angiographie après injection du produit de contraste dans les deux artères carotidiennes et une des artères vertébrales.

Pour garantir la comparabilité des résultats, les examens ont été accomplis le plus simultanément possible, l'intervalle entre l'un et l'autre des examens n'excédant pas trois heures.

\section{Observations}

Au total, 16 malades classifiés cliniquement en état de coma dépassé, ont fait l'objet de cette étude.

Tous étaient à température normale ou légèrement subnormale ; le rythme cardiaque était régulier et la tension artérielle à la limite inférieure de la normale, mais le débit urinaire était encore satisfaisant. Au point de vue neurologique, tous les réflexes crâniens étaient abolis, mème la réaction aux stimulations douloureuses. La respiration spontanée étant 309 
TABLEAU I

RÉSUMÉ DES RÉSULTATS DE LA FLUOROSCOPIE RÉTINIENNE ET DE L'ANGIOGRAPHIE CÉRÉBRALE CHEZ 16 MALADES

\begin{tabular}{|c|c|c|c|c|}
\hline $\begin{array}{l}\text { cas } \\
\text { no. }\end{array}$ & $\begin{array}{l}\text { Age at } \\
\text { sexe }\end{array}$ & diagnostic & $\begin{array}{l}\text { fuoroscopie } \\
\text { rétinienne }\end{array}$ & $\begin{array}{l}\text { angiographie } \\
\text { cérébrale }\end{array}$ \\
\hline 1 & $\begin{array}{c}59 \text { ans } \\
9\end{array}$ & $\begin{array}{l}\text { fracture du crâne } \\
\text { hématome épidural et } \\
\text { sousdural }\end{array}$ & $\begin{array}{l}0 \text { fluorescence à } \\
1 \text { minute }\end{array}$ & $\begin{array}{l}0 \text { circulation intra- } \\
\text { crănienne }\end{array}$ \\
\hline 2 & $\begin{array}{c}59 \text { ans } \\
9\end{array}$ & $\begin{array}{l}\text { hémorragie sousarach- } \\
\text { noidienne }\end{array}$ & $\begin{array}{l}0 \text { fluorescence à } \\
1 \text { minute }\end{array}$ & $\begin{array}{l}0 \text { circulation intra- } \\
\text { cranienne }\end{array}$ \\
\hline 3 & $\begin{array}{c}5 \text { ans } \\
6\end{array}$ & $\begin{array}{l}\text { fracture du crâne } \\
\text { hematome sousdural }\end{array}$ & $\begin{array}{l}0 \text { fluorescence à } \\
1 \text { minute }\end{array}$ & $\begin{array}{l}0 \text { circulation intra- } \\
\text { crânienne }\end{array}$ \\
\hline 4 & $\begin{array}{c}38 \text { ans } \\
d^{*}\end{array}$ & fracture du crâne & $\begin{array}{l}0 \text { fluorescence à } \\
1 \text { minute }\end{array}$ & $\begin{array}{l}0 \text { circulation intra- } \\
\text { crấnienne }\end{array}$ \\
\hline 5 & $\underset{d}{79}$ ans & $\begin{array}{l}\text { fracture du crâne } \\
\text { hématome sousdural }\end{array}$ & $\begin{array}{l}0 \text { fluorescence à } \\
1 \text { minute }\end{array}$ & $\begin{array}{l}0 \text { circulation intra- } \\
\text { crânienne }\end{array}$ \\
\hline 6 & $\begin{array}{c}73 \text { ans } \\
0\end{array}$ & $\begin{array}{l}\text { fracture du crâne } \\
\text { codème cérébral }\end{array}$ & $\begin{array}{l}0 \text { fluorescence à } \\
1 \text { minute }\end{array}$ & $\begin{array}{l}0 \text { circulation intra- } \\
\text { crânienne }\end{array}$ \\
\hline 7 & $\begin{array}{c}59 \text { ans } \\
6\end{array}$ & $\begin{array}{l}\text { fracture du crâne } \\
\text { contusion cérébrale } \\
\text { hématome cérébral }\end{array}$ & $\begin{array}{l}0 \text { fuorescence à } \\
1 \text { minute }\end{array}$ & $\begin{array}{l}0 \text { circulation intra- } \\
\text { crânienne }\end{array}$ \\
\hline 8 & $\begin{array}{c}57 \text { ans } \\
q\end{array}$ & $\begin{array}{l}\text { fracture du crâne } \\
\text { cedème cérébral }\end{array}$ & $\begin{array}{l}\text { fluorescence après } \\
25 \text { seconddes }\end{array}$ & $\begin{array}{l}\text { circulation infra- } \\
\text { tentorielle }\end{array}$ \\
\hline 9 & $\begin{array}{c}46 \text { ans } \\
\delta\end{array}$ & $\begin{array}{l}\text { hémorragie sousarach- } \\
\text { nol̈dienne }\end{array}$ & $\begin{array}{l}\dot{0} \text { fluorescence à } \\
1 \text { minute }\end{array}$ & $\begin{array}{l}\text { O circulation intra- } \\
\text { crânienne }\end{array}$ \\
\hline 10 & $\begin{array}{c}44 \text { ans } \\
d\end{array}$ & $\begin{array}{l}\text { fracture du crâne } \\
\text { hémorragie sousarach- } \\
\text { noỉdienne }\end{array}$ & $\begin{array}{l}0 \text { fluorescence à } \\
1 \text { minute }\end{array}$ & $\begin{array}{l}\text { O circulation intra- } \\
\text { crânienne }\end{array}$ \\
\hline 11 & $\begin{array}{c}10 \text { ans } \\
9\end{array}$ & $\begin{array}{l}\text { malformation artério- } \\
\text { veineuse cérébrale }\end{array}$ & $\begin{array}{l}\text { fluorescence après } \\
32 \text { secondes }\end{array}$ & $\begin{array}{l}0 \text { circulation intra- } \\
\text { crânienne }\end{array}$ \\
\hline 12 & $\begin{array}{c}53 \text { ans } \\
9\end{array}$ & $\begin{array}{l}\text { fracture du crâne } \\
\text { hématome sousdural }\end{array}$ & $\begin{array}{l}0 \text { fluorescence à } \\
1 \text { minute }\end{array}$ & $\begin{array}{l}0 \text { circulation intra- } \\
\text { crânienne }\end{array}$ \\
\hline 13 & $\begin{array}{c}67 \text { ans } \\
9\end{array}$ & $\begin{array}{l}\text { métastase cérébral } \\
\text { néo du côlon }\end{array}$ & $\begin{array}{l}0 \text { fluorescence } a \\
1 \text { minute }\end{array}$ & $\begin{array}{l}\text { o circulation intra- } \\
\text { crânienne }\end{array}$ \\
\hline 14 & $\begin{array}{c}25 \text { ans } \\
d\end{array}$ & $\begin{array}{l}\text { fracture du crâne } \\
\text { hématome épidural }\end{array}$ & $\begin{array}{l}0 \text { fluorescence } a \\
1 \text { minute }\end{array}$ & $\begin{array}{l}0 \text { circulation intra- } \\
\text { crânienne }\end{array}$ \\
\hline 15 & $\begin{array}{c}16 \text { ans } \\
\delta\end{array}$ & $\begin{array}{l}\text { fracture du crâne } \\
\text { contusion cérébrale }\end{array}$ & $\begin{array}{l}\text { fluorescence après } \\
25,4 \text { secondes }\end{array}$ & $\begin{array}{l}\text { circulation infra- } \\
\text { tentorielle }\end{array}$ \\
\hline 16 & $\begin{array}{c}73 \text { ans } \\
9\end{array}$ & hématome cérébral & $\begin{array}{l}\text { fluorescence après } \\
15 \text { secondes }\end{array}$ & $\begin{array}{l}\text { circulation intra- } \\
\text { crânienne mais sté- } \\
\text { nose de l'artère } \\
\text { vertébrale droite }\end{array}$ \\
\hline
\end{tabular}

\section{RÉSULTATS}

Le tableau I l'énumère la liste des cas inclus dans celte étude et présente l'ensemble des résultats. L'angiographie cérébrale a démontré l'arrèt de la circulation intra-crânienne chez 12 des malades et une circulation gravement réduite et uniquement infratentorielle chez trois malades.

La fluroscopie rétinienne pour sa part a égale- ment montré l'arrêt de la circulation intracrânienne par absence de fluorescence rétinienne chez 12 des comateux et a témoigné d'un retard séricux de la circulation par un temps de fluarescence de $25,0,25,4$ et 32,0 secondes chez les trois autres malades.

Le tableau II compare les résultats avec les deux méthodes et illustre bien la concordance entre les données de l'angiographie et celles de lobservation de la fluorescence. 
TABLEAU II

Comparaison des résultats

\begin{tabular}{|c|c|c|c|}
\hline & \multicolumn{3}{|c|}{ angiographie cérébrale } \\
\hline $\begin{array}{l}\text { fuoroscopie } \\
\text { rétinienne }\end{array}$ & $\begin{array}{c}\text { circulation } \\
\text { intra- } \\
\text { crânienne } \\
\text { normale }\end{array}$ & $\begin{array}{c}\text { circulation } \\
\text { infra- } \\
\text { tentorielle }\end{array}$ & $\begin{array}{l}\text { pas de } \\
\text { circulation } \\
\text { intra- } \\
\text { crânienne }\end{array}$ \\
\hline
\end{tabular}

\begin{tabular}{llll}
$\begin{array}{l}\text { fluorescence } \\
\text { rétinienne } \\
15 \text { secondes }\end{array}$ & 1 & 0 & 0 \\
$\begin{array}{l}\text { fluorescence } \\
\text { rétinienne } \\
\text { entre } 15 \text { et } \\
25 \text { secondes } \\
\text { fuorescence }\end{array}$ & 0 & 0 & 0 \\
rétinienne & & & \\
entre 25 et \\
$\begin{array}{l}60 \text { secondes } \\
\text { pas de fluo- } \\
\text { rescence à }\end{array}$ & 0 & 2 & 1 \\
1 minute & 0 & 1 & 11 \\
total & 1 & 3 & 12 \\
\hline
\end{tabular}

\section{Discussion}

Le temps de circulation bras-rétine normal peut varier de 5 à 15 secondes (moyenne : 12 secondes) selon les circonstances de l'examen ; le sang circule alors sans obstacle du cour à la tête. Un temps de circulation bras-rétine supérieur à 15 secondes indique un ralentissement du courant sanguin et, prolongé à plus de 25 secondes, il indique vraisemblablement une obliteration de l'artère carotidienne interne qui force le sang à passer par des anastomoses de l'artère carotidienne externe. La perfusion du cerveau étant ainsi gravement réduite, l'ischémie et la nécrose du tissu nerveux en résulteront bientôt.

Dans une étude de 50 cas de coma dépassé. Mantz et ses collègues ${ }^{3}$ ont trouvé la circulation carotidienne interne arrèté au niveau du siphon quand le temps de circulation bras-rétine a été supérieur à 22 secondes. Pour garder une marge de sécurité suffisante et pour éliminer des possibilités d'erreur, comme par exemple des obstacles artériels du bras ou du cour, une chute de la pression sanguine ou une erreur de chronométrage, on a proposé un allongement du temps supérieur à 30 secondes comme un critère de mort cérébrale. Jahro 4 a donné 28 secondes comme une limite absolue et Arnold ${ }^{5}$ a donné le chiffre de 30 secondes.
Ayant exclu les mêmes possibilités d'erreur dans notre travail, nous avons démontré angiographiquement qu'un temps de circulation bras-rétine supérieur à 25 secondes correspond soit à l'arrêt de la totalité de la circulation intracrânienne, soit à l'arrêt de la portion supratentorielle de celle-ci, entrainant la mort du cerveau par ischémie et nécrose.

Le malade numéro 16 mérite un commentaire. L'angiographie cérébrale a montré une circulation normale, à l'exception d'une sténose de l'artère vertébrale gauche. Ce résultat a confirmé ceux de la fluoroscopie qui a donné un temps de 15 secondes. Ce malade n'était pas en état de mort cérébrale au moment des investigations.

Pour bien étalonner la méthode à la fluorescéine de façon simple et rapide, nous avons choisi à volonté le pli du coude comme point d'injection. Ce site est un niveau anatomiquement bien défini, où il est facile de trouver une veine ; un cathéter veineux central, au contraire. commande un examen radiologique supplémentaire pour sa localisation précise.

La fluoroscopie rétinienne a cependant ses limitations dont il faut tenir compte. Le temps de circulation bras-rétine peut s'allonger sans qu'il n'existe de défaillance circulatoire cérébrale : une anomalie vasculaire et/ou un obstacle au courant sanguin entre le site d'injection et l'artère ophtalmique, provoqueront un allongement. Une hypothermie accidentelle entrainant un ralentissement du courant sanguin par réduction du débit cardiaque donnera le mème effet. Une erreur d'injection du colorant ou une faute de la mesure du temps pourra également fausser le résultat.

De plus, il faut préciser que l'information fournie est uniquement indicative, tandis que la preuve formelle de l'arrèt de la circulation intra-crânienne est donnée par l'angiographie cérébrale.

\section{Conclusion}

Tout en reconnaissant les limitations de la fluoroscopie rétinienne, nous croyons qu'elle peut s'avérer fort utile pour vérifier la présence d'ischémie cérébrale chez le comateux. A cet égard, les données de la fluoroscopie rétinienne sont, dans ce travail, comparables à celles de l'angiographie cérébrale. Nous considérons un temps de circulation bras-rétine supérieur à 25 secondes comme un critère de mort cérébrale. Ce test simple est facilement applicable et nous apparaît utile pour orienter le thérapeute devant un coma dépassé. 
Résumé

La fluoroscopie rétinienne, avec mesure du temps de circulation bras-rétine, permet d'évaluer l'état de la perfusion du cerveau. Au cours d'ischémie cérébrale par défaillance perfusionnelle du cerveau, le temps de circulation bras-rétine est prolongé de plusieurs secondes et la fluorescence rétinienne est retardée, ou même abolie.

Dans cette étude, 16 malades cliniquement classifiés en état de coma dépassé, ont été examinés par fluoroscopie rétinienne et par angiographie cérébrale. Les résultats obtenus avec les deux métbodes sont totalement concordants. La méthode à la fluorescéine est simple, rapide, et fournit des informations utiles au sujet de la circulation intra-crânienne. Elle permet de diagnostiquer la mort cérébrale avec une plus grande certitude.

Nous concluons que l'on peut considérer la circulation intracrânienne à toute fin pratique comme arrètée si la fluorescence rétinienne n'apparaît qu'après un délai de 25 secondes. Nous proposons donc qu'un temps de circulation bras-rétine supérieur à 25 secondes soit considéré comme un critère de mort cérébrale.

\section{SUMMaRY}

Retinal fluoroscopy with measurement of the arm to retina circulation time can be used to evaluate the blood supply to the brain. When the brain tissue suffers from ischaemia as a result of circulatory failure, the arm to retina circulation time will be prolonged.

Sixteen patients in deep coma resulting from serious cerebral disease were examined to verify cerebral ischaemia. The results of retinal fluoroscopy and cerebral angiography agreed. We find that retinal fluoroscopy is a simple and reliable method that can be used to detect brain death in comatose patients treated by automatic ventilation. We conclude that the main cerebral circulation can be regarded as occluded if the retinal fluorescense does not appear within 25 seconds after antecubital intravenous injection of fluoresceine.

We suggest that the arm to retina circulation time exceeding 25 seconds may be used as a criterion of death.

\section{BIBLIOGRAPHIE}

1. Davio. N.J., Salto, Y. \& Heyman, A. Arm to retina fluorescein appearance time. Arch. Neurol. 5: 165-170(1961).

2. Gilland, O., et al. Arm-retina fluorescein circulation time in carotid obstruction. Acta Neurol. Scand. 319-333 (1965).

3. MANTZ, J.M., et al. L'ail dans le diagnostic de la mort cérébrale. Ann. Anesth. Fr. 3:95-100 (1974).

4. Jahro, L., Weckman, N. \& AIKAS, T. Arm-retina fuorescein time in cerebrovascular disorders. Duodecim, 48: 183-191 (1968).

5. ARnold, H. Hirntod. Nervenarzt 47: 529-537 (1976). 